\title{
Bright single photon emission from a quantum dot in a circular Bragg grating microcavity
}

\author{
Serkan Ateş, Luca Sapienza, Marcelo Davanço, Antonio Badolato, and Kartik Srinivasan
}

\begin{abstract}
Bright single photon emission from single quantum dots in suspended circular Bragg grating microcavities is demonstrated. This geometry has been designed to achieve efficient $(>50 \%)$ single photon extraction into a near-Gaussian shaped far-field pattern, modest $(\approx 10 x)$ Purcell enhancement of the radiative rate, and a spectral bandwidth of a few nanometers. Measurements of fabricated devices show progress towards these goals, with collection efficiencies as high as $\approx 10 \%$ demonstrated with moderate spectral bandwidth and rate enhancement. Photon correlation measurements are performed under abovebandgap excitation (pump wavelength $=780 \mathbf{n m}$ to $820 \mathbf{n m}$ ) and confirm the single photon character of the collected emission. While the measured sources are all antibunched and dominantly composed of single photons, the multi-photon probability varies significantly. Devices exhibiting tradeoffs between collection efficiency, Purcell enhancement, and multi-photon probability are explored and the results are interpreted with the help of finitedifference time-domain simulations. Below-bandgap excitation resonant with higher states of the quantum dot and/or cavity (pump wavelength $=860 \mathrm{~nm}$ to $900 \mathrm{~nm}$ ) shows a near-complete suppression of multi-photon events and may circumvent some of the aforementioned tradeoffs.
\end{abstract}

\section{INTRODUCTION}

Single epitaxially-grown $\operatorname{In}_{x} \mathrm{Ga}_{1-x}$ As quantum dots (QDs) have generated significant interest as potentially bright and stable single photon sources for quantum information processing applications [1], [2], [3]. In principle, the maximum achievable single photon rate is limited only by the spontaneous emission lifetime $\left(\tau_{\mathrm{sp}}\right)$ of the $\mathrm{QD}$, so that for a single InGaAs QD with a typical $\tau_{\mathrm{sp}} \approx 1 \mathrm{~ns}$ in bulk, a single photon rate $R \approx 1 / \tau_{s p}$ approaching $1 \mathrm{GHz}$ may be possible. Still faster rates can be achieved through Purcell enhancement of the QD radiative rate [4]. In practice, however, the available single photon rates are not necessarily limited by the generation rate, but by the efficiency with which the single photons are funneled into a useful collection channel (e.g., low divergence angle far-field emission). This well-known problem [5], [6], [7] is fundamentally due to the high-refractive index contrast between the GaAs material in which the QD is embedded (refractive index $n \approx 3.4$ ) and the surrounding air $(n=1)$. This strongly limits the amount of free-space emission exiting the

S. Ateş, L. Sapienza, M. Davanço, and K. Srinivasan are with the Center for Nanoscale Science and Technology, National Institute of Standards and Technology, Gaithersburg, MD 20899-6203, USA (e-mail: serkan.ates@ nist.gov; kartik.srinivasan@ nist.gov)

A. Badolato is with the Department of Physics and Astronomy, University of Rochester, Rochester, NY 14627

S. Ateş and M. Davanço are also with the Maryland Nanocenter, University of Maryland, College Park, MD 20742

L. Sapienza's present address is School of Engineering and Physical Sciences, Heriot-Watt University, Edinburgh, EH14 4AS, UK semiconductor because of total internal reflection. Even with high numerical aperture (NA) optics, a maximum theoretical collection efficiency $<1 \%$ is expected.

This issue has been addressed by a number of groups. Solid immersion lenses [8], [9] provide a broadband and versatile approach to directly increase the collection efficiency. Alternatively, nanofabricated photonic structures such as optical microcavities [10], [11], [12] and waveguides[13], [14] provide confined or guided modes into which the QD can radiate. Ideally, the QD will dominantly radiate into a single mode of the structure (high spontaneous emission coupling factor $\beta$ ), which can then be efficiently out-coupled in the far-field depending on its emission pattern and divergence angle. Recent work [10], [13] has demonstrated efficiencies in excess of $50 \%$ into the first optical element of a fluorescence microscopy setup. The microcavity and waveguide approaches each offer their own advantages - microcavities supporting high quality factor $(Q)$ optical modes provide radiative rate enhancement, albeit over a narrow spectral band (the width of the cavity mode), while waveguides are broadband devices but typically do not offer rate enhancement (unless they operate in a slow-light regime). Broadband operation avoids the need for precise spectral alignment of the QD emission line to a photonic resonance and allows for efficient spectroscopy of multiple spectrally separated states of a single QD. On the other hand, radiative rate enhancement increases the maximum rate at which single photons can be generated by the QD, and can improve the indistinguishability of the photons [15], [16].

In ref. [17], we proposed and demonstrated a microcavity geometry for efficient extraction of photons from single QDs. This 'bullseye' geometry, shown in Fig. 1, consists of a series of partially etched grooves in a suspended GaAs membrane containing a layer of InAs QDs. In comparison to many of the bright single photon geometries demonstrated thus far [11], [10], [13], which are based on vertically-oriented micropillars or nanowires, the bullseye geometry is planar, thereby requiring a relatively simple fabrication process. The fundamental optical mode of this cavity has a small volume $V_{\text {eff }} \approx 1.3(\lambda / n)^{3}$, but a relatively low $Q=200$. This allows for both modest Purcell enhancement (maximum value $12 \times$ predicted in theory) and moderate spectral bandwidth (few $\mathrm{nm}$ for QDs emitting at $\lambda \approx 940 \mathrm{~nm}$ ). In addition, the geometry supports a far-field emission pattern that is near-Gaussian and predominantly directed vertically above the sample surface. Collection efficiencies higher than $50 \%$ (for NA $\gtrsim 0.42$ ) are predicted theoretically, and a measured efficiency of $10 \%$ was reported in ref. [17].

In this paper, we present further developments of the bulls- 


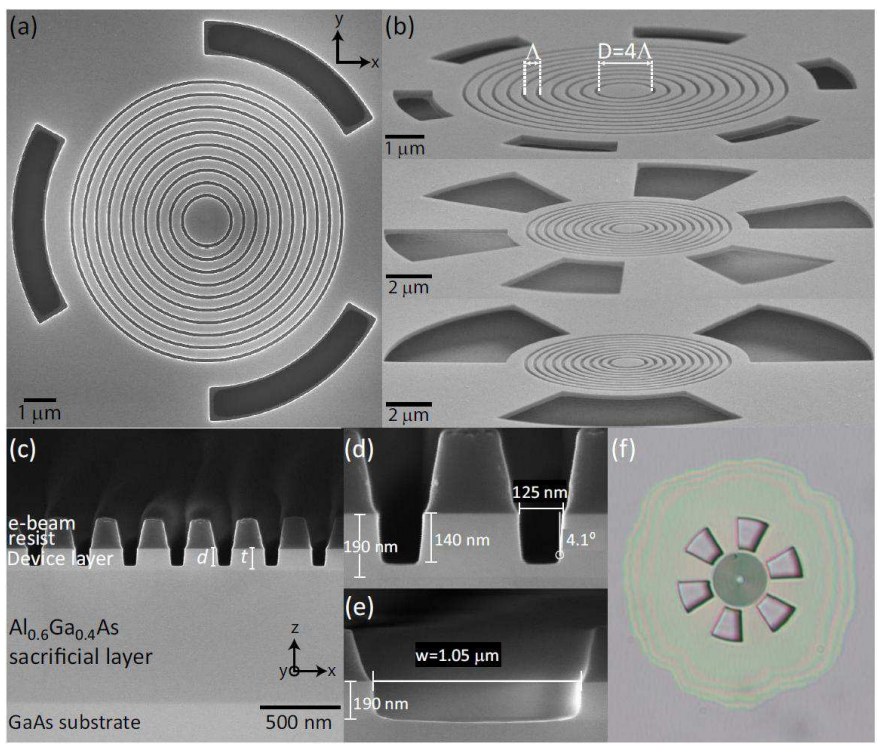

Fig. 1. (a) Top view and (b) angled view scanning electron microscope (SEM) images of the bullseye cavities with different undercut access geometries. (c)(e) Cross-sectional SEM images of the bullseye cavities after the GaAs dry etch but before the HF undercut. The grating trenches are etched to a depth $d$ that is below the QD-containing layer (located at $t / 2$ ) but smaller than the GaAs thickness $(t)$. In comparison, the access areas outside of the grating are fully etched through the GaAs layer, allowing the devices to be undercut. (f) Optical microscope image of a fully processed device.

eye cavity, with a focus on better understanding its potential in single photon source applications. A key question left unanswered in ref. [17] is the degree to which multi-photon probability is suppressed in the out-coupled emission from the device, given the fact that the QD lines are situated on top of a broad cavity mode which also effectively out-couples other (non single photon) emission from multi-excitonic and hybridized QD-wetting layer states [18], [19]. Through photon correlation measurements of a series of devices under abovebandgap excitation (pump wavelength $=780 \mathrm{~nm}$ to $820 \mathrm{~nm}$ ), we observe tradeoffs between collection efficiency, Purcell enhancement, and multi-photon probability. These tradeoffs, and the reduction in collection efficiency and Purcell enhancement relative to the maximum predicted values, are interpreted with the help of finite-difference time-domain simulations which focus on the role of variations in the grating geometry and QD position and orientation in influencing device performance. The results indicate that if adequate control of the QD position [20] and grating dimensions can be exercised, the device behavior may be tuned according to the application at hand (for example, limiting Purcell enhancement in exchange for single photon purity). Ideally, such tradeoffs would not be necessary, and as a first step towards improving this, we pursue below-bandgap excitation resonant with higher states of the QD and/or cavity (pump wavelength $=860 \mathrm{~nm}$ to 890 $\mathrm{nm})$. These measurements show a near-complete suppression of multi-photon events and may circumvent some of the aforementioned limitations.

\section{DEVICE DESIGN}

Our nanophotonic structure (Fig. 1) consists of a circular dielectric grating with radial period $\Lambda$ surrounding a central region of radius $R=2 \Lambda$, produced on a suspended GaAs membrane. The GaAs slab of thickness $t$ supports single TE and TM polarized modes (electric or magnetic field parallel to the slab, respectively) at wavelengths near $980 \mathrm{~nm}$. The grating is composed of partially etched circular trenches that have width $w$, depth $d(t / 2<d<t)$, and are radially spaced by a period $\Lambda$. Quantum dots are grown at the center of the GaAs membrane $(z=0)$, and are located randomly in the $x y$ plane.

This 'bullseye' geometry favors extraction of emission from QDs in the central circular region, which would otherwise be trapped inside the GaAs membrane. Indeed, a QD located in the central region radiates dominantly into slab-guided waves due to total internal reflection at the semiconductorair interface. The role of the circular grating is to scatter such guided waves into free-space, preferentially upwards, towards the collection optic. Effective light extraction can be achieved via a second order Bragg grating for the slab modes (with period equal to the guided wave wavelength), which provides a first order diffraction perpendicular to the slab plane [21], [22]. In [22], linear gratings were developed to provide efficient coupling between free-space beams and planar waveguides modes. In our case, a circular grating geometry was a more natural choice because QD emission in the GaAs slab was expected to be cylindrically symmetric. We point out that similar circular geometries have been employed for enhanced light extraction from light emitting diodes [23], and for demonstrating annular Bragg lasers [24].

The grating period $\Lambda$ was chosen to approximately satisfy the second-order Bragg condition, $\Lambda=\lambda_{Q D} / n_{T E}$, where $n_{T E}$ is the GaAs slab TE mode effective index. Because this formula is valid in the limit of a weak grating, which is not the case here (as the grating's etch depth is a considerable fraction of the slab thickness), we use it as a starting point in an optimization procedure which determines the correct period.

Partial reflections at the grating towards the center of the geometry lead to the formation of cavity resonances such as shown in Fig. 22(a), where the particular orientation of the cavity mode is determined by the orientation of the radiating dipole ( $x$ direction in this case). The large index contrast at the trenches leads to strong reflections and out-of-slab-plane scattering at the semiconductor-air interfaces, as apparent in Fig. 2(b). Resonances are centered at wavelengths determined by the radius of the central region and the grating geometry itself, which determines the overall phase of the reflected waves. Figure 2 (c) shows the evolution of the resonance center wavelength for three varying grating periods $\Lambda$. In addition to the trench spacing, large differences in propagation constants in the semiconductor and air regions of the grating produce significant resonance spectral shifts with small variations in trench width and depth.

The trench depth $d$ has a strong influence on the quality factor $(Q)$ and vertical light extraction, as incomplete spatial overlap between a trench and an incident slab-bound wave 


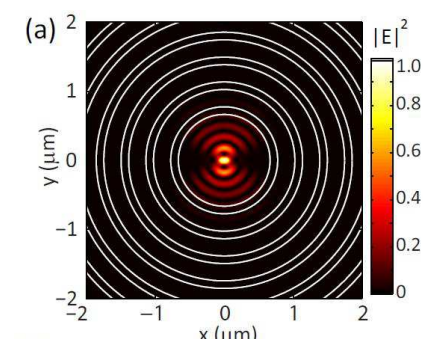

(c)

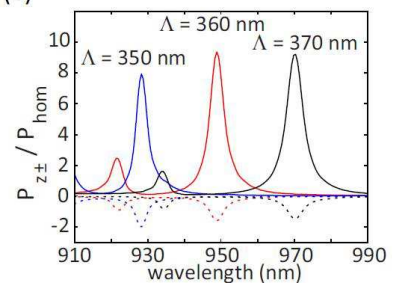

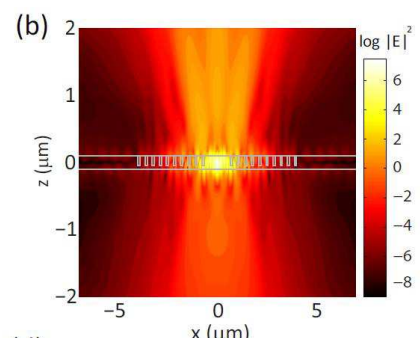

(d)

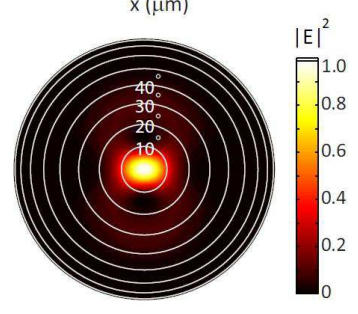

Fig. 2. Electric field intensity in the (a) $x y$ and (b) $x z$ planes (log scale). (c) Calculated vertically extracted power as a function of wavelength, normalized to the homogeneous medium electric dipole power $P_{\text {hom }}$ for $d=0.70 t$. Continuous lines: upwards $(+z)$ extraction; dotted: downwards $(-z)$. (d) Farfield polar plot for the cavity mode with $\Lambda=360 \mathrm{~nm}$.

leads to both coupling to radiating waves and lower modal reflectivity. Preferential upwards vertical extraction results from the grating asymmetry, as evidenced in Fig. 2 (c). This tradeoff between the quality factor $Q$, power extraction and power extraction asymmetry, and far-field collimation due to variation in the trench depth is discussed further in Section IV-A. We note that our suspended, asymmetric grating approach limits radiation into the substrate without the need to oxidize the AlGaAs, bond the grating to a low index layer[24], or utilize a deeply etched geometry [10], [13].

In Fig. 2(c), the maximum upwards extracted powers are $\approx 10 \times P_{\text {hom }}$ ( $P_{\text {hom }}$ is the emitted power for an electric dipole in a homogeneous GaAs medium), an indication of Purcell radiation rate enhancement due to the cavity [25], [26]. Indeed, for the $\Lambda=360 \mathrm{~nm}$ structure the enhancement $F_{p}$ at the maximum extraction wavelength $\left(\lambda_{c}=948.9 \mathrm{~nm}\right)$ is $F_{p}=P_{\text {tot }} / P_{\text {Hom }}=11.0$, where $P_{\text {tot }}$ is the total radiated power in all directions. This resonance has $Q=200$, and its effective mode volume, calculated from the field distribution, is $V_{\text {eff }}=1.3\left(\lambda_{c} / n\right)^{3}$ ( $n$ is the GaAs refractive index) [17]. Thus, despite the relatively low quality factor, the mode volume can be sufficiently small to produce a significant Purcell factor.

Finally, the far-field of the emitted radiation is highly directional, as evidenced in Fig. 2(d). Here, the emitted field for a dipole located at the bullseye center is nearly gaussian, and mostly contained within a $20^{\circ}$ half-angle. Indeed, in [17], we show that $\approx 53 \%$ of the total emission can be collected with a NA=0.42 optic, while collection superior to $80 \%$ of the total emission can be achieved with NA $>0.7$. The dependence of the emitted field's spatial distribution on geometric parameters is non-trivial, however, and is discussed further in Section IV-A

\section{Design Procedure}

The design process used to generate Fig. 2 above and the results to be discussed in Section IV-A consisted of a series

of finite-difference time-domain simulations that sought to maximize vertical light extraction near the QD's expected sshell emission $\left(\lambda_{Q D} \approx 940 \mathrm{~nm}\right)$, by varying $\Lambda, t$, and $w$. The structures were excited with a horizontally oriented electric dipole at the bullseye center $(x=0, y=0)$, representing an optimally placed QD. Total radiated power, steady-state upwards and downwards emission, and electromagnetic fields were then recorded at several wavelengths. The dipole orientation was assumed to be aligned along the $x y$ plane, exciting only TE slab waves (self-assembled InAs QDs are expected to have electric dipole on the GaAs slab plane).

Steady-state fields at a surface just above the GaAs slab were used to calculate far-field patterns as in Fig. 22(d). The power $P_{\text {coll }}$ collected by an optic of numerical aperture NA was calculated by integrating the far-field pattern over the appropriate angular range, and multiplying by the power radiated upwards.

\section{EXPERIMENTAL RESUlts UnDER ABOVE-BAND EXCITATION}

\section{A. Experimental Setup}

Figure 3 shows a detailed schematic view of several experimental setups used within this work. The sample was mounted on a cold finger and placed in a continuous flow liquid $\mathrm{He}$ cryostat that sits on a two-axis nano-positioning stage. Two different excitation sources were used to optically excite the QDs: (i) a CW laser diode $(780 \mathrm{~nm})$ for aboveband excitation, (ii) a tunable pulsed fiber laser $(820 \mathrm{~nm}$ to $950 \mathrm{~nm}$ ) for quasi-resonant excitation. Spectral properties of the QD emission were investigated via a low-temperature micro-photoluminescence ( $\mu$-PL) setup (Fig 3 (a)), where a single microscope objective $(\mathrm{NA}=0.42)$ was used for both the illumination of the sample and the collection of the emission. The collected signal is directed to a $500 \mathrm{~mm}$ focal length spectrometer either to record an emission spectrum with a $\mathrm{Si}$ Charge-Coupled Device (CCD) or to filter a single emission line for further investigations. For the second-order correlation function $g^{(2)}(\tau)$ measurements, the spectrally filtered emission (with a linewidth $\approx 70 \mathrm{pm} \approx 100 \mu \mathrm{eV}$ ) is directed to a Hanbury-Brown and Twiss (HBT) type interferometer that consists of a 50/50 non-polarizing beamsplitter (NPBS) and two high quantum efficiency (QE) single-photon counting avalanche diodes (SPADs), as shown in Fig. 3 (b). The SPADs (peak $\mathrm{QE}=73 \%$ at $700 \mathrm{~nm}$ and $\mathrm{QE}=28 \%$ at $980 \mathrm{~nm}$ ) were connected to a time-correlated single-photon counting module to create a histogram of photon detection events. The dynamics of a single QD emission was measured by using a timecorrelated single-photon counting (TCSPC) technique, which relies on measuring the time delay between an excitation pulse and detection of an emitted photon by using a fast SPAD (Fig. 3(c), timing jitter $=50 \mathrm{ps}, \mathrm{QE}=3 \%$ at $940 \mathrm{~nm}$ ). Finally, for first-order field correlation function $g^{(1)}(\tau)$ measurements, a Michelson interferometer was attached to the output of the spectrometer. As shown in Fig. 3 d), the setup consists of a 50/50 NPBS and two retro-reflectors, one of which is mounted on a linear stage to provide a coarse tunable optical delay up to $4 \mathrm{~ns}$ and the other retro-reflector is attached on a 


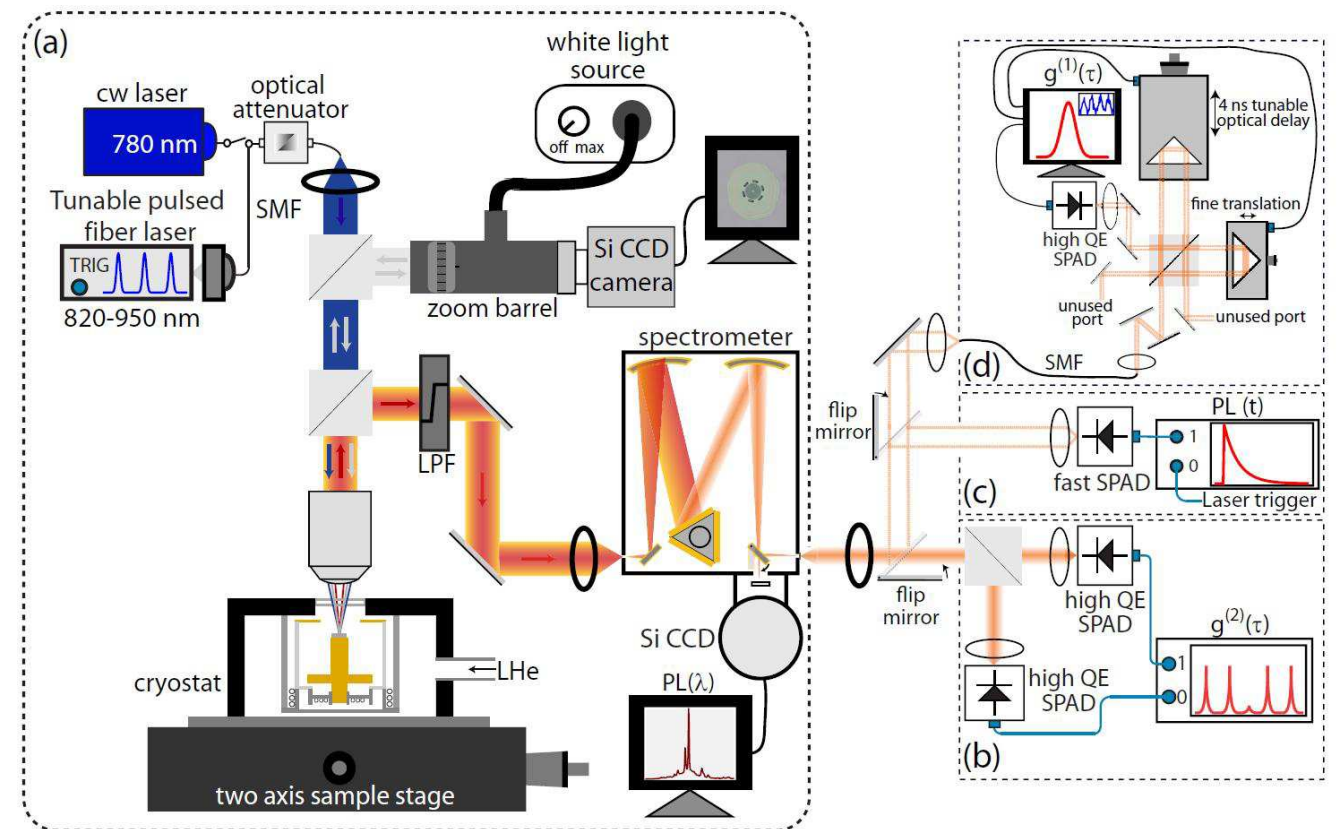

Fig. 3. Schematic of the experimental setup: (a) Confocal $\mu$-PL, (b) Hanbury-Brown and Twiss photon correlation setup, (c) time-resolved PL, and (d) Michelson interferometer. SMF: single mode fiber, LPF: long pass filter

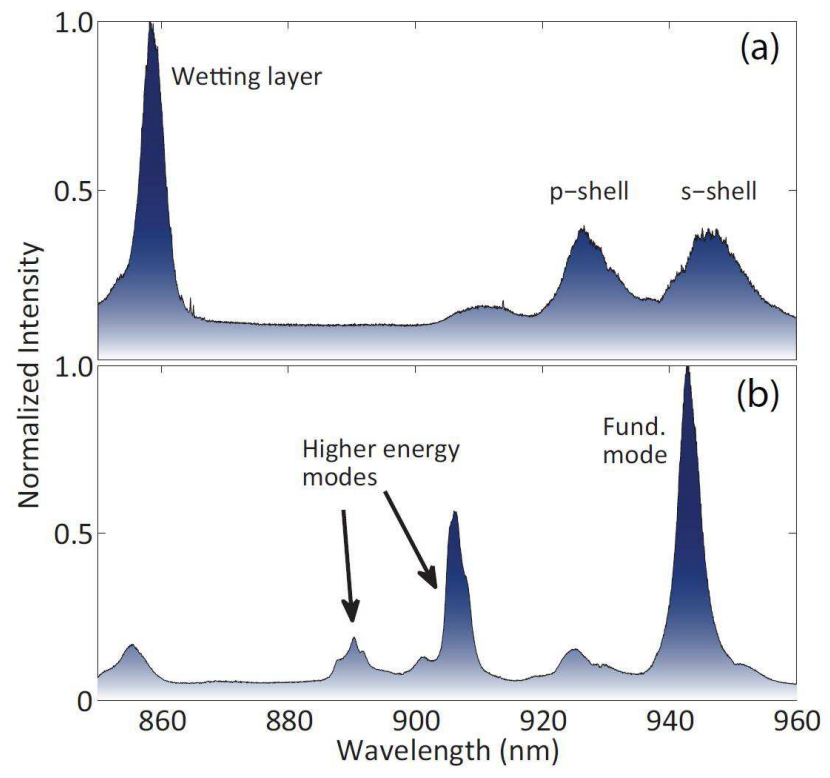

Fig. 4. (a) Low temperature broad range PL spectrum of QD-ensemble under high excitation power conditions. S-shell, p-shell and wetting layer emission of the ensemble are observed. (b) Low temperature broad range PL spectrum of a Bullseye device which shows the fundamental mode and the higher energy modes of the cavity.

piezo actuator for a fine delay. The interference fringes were recorded by a SPAD, which was attached to one output port of the interferometer.

\section{B. Collection efficiency, time-resolved photoluminescence, and photon antibunching}

Initial characterization of the bare (unprocessed) QD sample has been done under strong pump powers with an excitation energy above the GaAs bandgap $(780 \mathrm{~nm})$, which excites all QDs within the excitation spot. Figure 4a) shows a low temperature wide range $\mu$-PL spectrum of the QD ensemble, where clear s-shell and p-shell emission of the ensemble are observed around $945 \mathrm{~nm}$ and $927 \mathrm{~nm}$, respectively. The sharper peak around $858 \mathrm{~nm}$ is attributed to the wetting layer of the sample. In order to limit the number of QDs coupled to a mode, the bullseye devices are fabricated in a low QD density $\left(\approx 1 \mu \mathrm{m}^{-2}\right)$ portion of the wafer and are designed to have their fundamental cavity mode spectrally aligned with the tail of the s-shell of the QD ensemble. We focus on three devices, named as BE1, BE2, and BE3, which have the same nominal grating period $\Lambda$ and central diameter $D$, but differ due to slight variations in fabrication across the chip (trench width and depth) and perhaps most importantly, due to the varying QD location and orientation within the devices (which is random). Figure 4(b) shows a similar wide range high power $\mu$-PL spectrum of device BE1, which clearly shows the fundamental mode (FM) and the well-separated higher energy modes of the cavity. The quality factor of the FM is measured from the ratio of the emission energy to the linewidth as $Q=200$.

Figure 5(a) shows a low temperature $(\mathrm{T}=10 \mathrm{~K}) \mu$-PL spectrum of BE1 taken under weak pulsed excitation of the GaAs $(\lambda=820 \mathrm{~nm})$. This device is the same as that presented in ref. [17], and displays bright emission lines labeled $X_{1}$ and $\mathrm{X}_{2}$ that sit on top of a broad background due to the cavity mode emission, which is thought to be mainly fed by the multi-exciton states of several nearby QDs [18]. Both $\mathrm{X}_{1}$ and $\mathrm{X}_{2}$ emission lines have almost $10 \%$ collection efficiency $\eta$ at their saturation powers, and time-resolved PL measurements performed on the $\mathrm{X}_{1}$ line resulted in a lifetime as fast as $360 \pm 6 \mathrm{ps}$, which is likely indicative of Purcell rate enhancement, as discussed later. In ref. [17], the characteristics of the $\mathrm{X}_{1}$ and $\mathrm{X}_{2}$ emission lines were investigated through pump-power-dependent and temperaturedependent PL measurements. Here, we focus on the single photon nature of the emission lines by measuring photon 

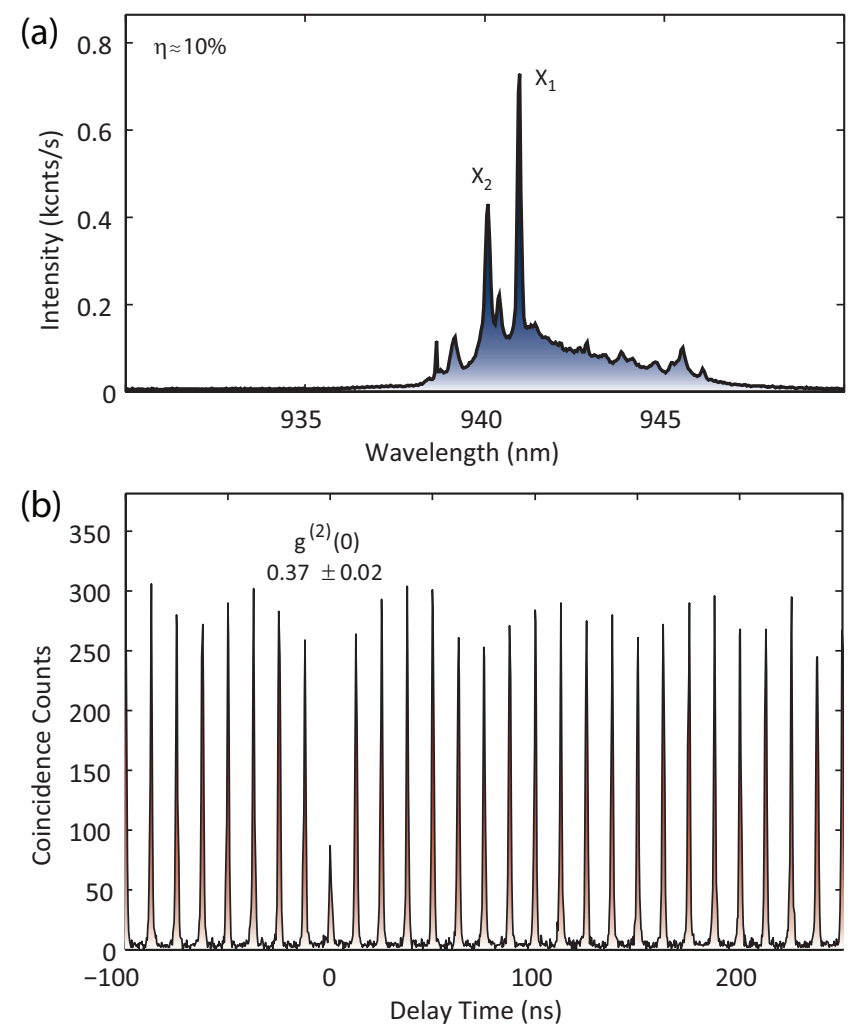

Fig. 5. (a) A typical low power $\mu$-PL spectrum of BE1 under pulsed GaAs excitation at a temperature $\mathrm{T}=10 \mathrm{~K}$. Lines $X_{1}$ and $X_{2}$ at $940 \mathrm{~nm}$ and $941 \mathrm{~nm}$, respectively, are states of a single QD that yield a collection efficiency $\eta$ of $10 \%$. (b) The result of second-order correlation function measurement on the $X_{1}$ line. A clear suppression of the central peak with $g^{(2)}(\tau)=0.37<0.5$ proves the single-photon nature of the collected emission.

statistics through the HBT interferometer setup (Fig. 3 b) under similar excitation conditions as in the previous work. Figure 5 (b) shows the result of an intensity autocorrelation measurement performed on the spectrally filtered $X_{1}$ line at its saturation power. A clear suppression of the peak at zero time delay to a value of $g^{(2)}(\tau)=0.37<0.5$ is seen [27], verifying that the measured line is originated from a single QD [2]. The deviation from the ideal value of $g^{(2)}(\tau)=0$ for a single quantum emitter is most likely related to the uncorrelated background emission coupled to the cavity mode at the same frequency.

In Fig. 5, bright single photon generation from a single QD with a large collection efficiency and fast fluorescence decay time have been demonstrated. However, the autocorrelation measurements resulted in a clear background at the QD emission frequency due to contributions from other states resonant with the cavity mode emission. This suggests a potential trade-off between either (or both) high extraction efficiency and single photon purity of the collected signal or fast fluorescence decay time and purity of the collected signal. To further investigate this, two more devices (named as BE2 and BE3) were studied in detail. Figure 6(a) shows a low power emission spectrum of device BE2 taken under aboveband pulsed excitation of the GaAs at $\mathrm{T}=10 \mathrm{~K}$. Similar to the device BE1, this device also shows two bright states named as excitonic (X) and bi-excitonic (XX) emission, which sit
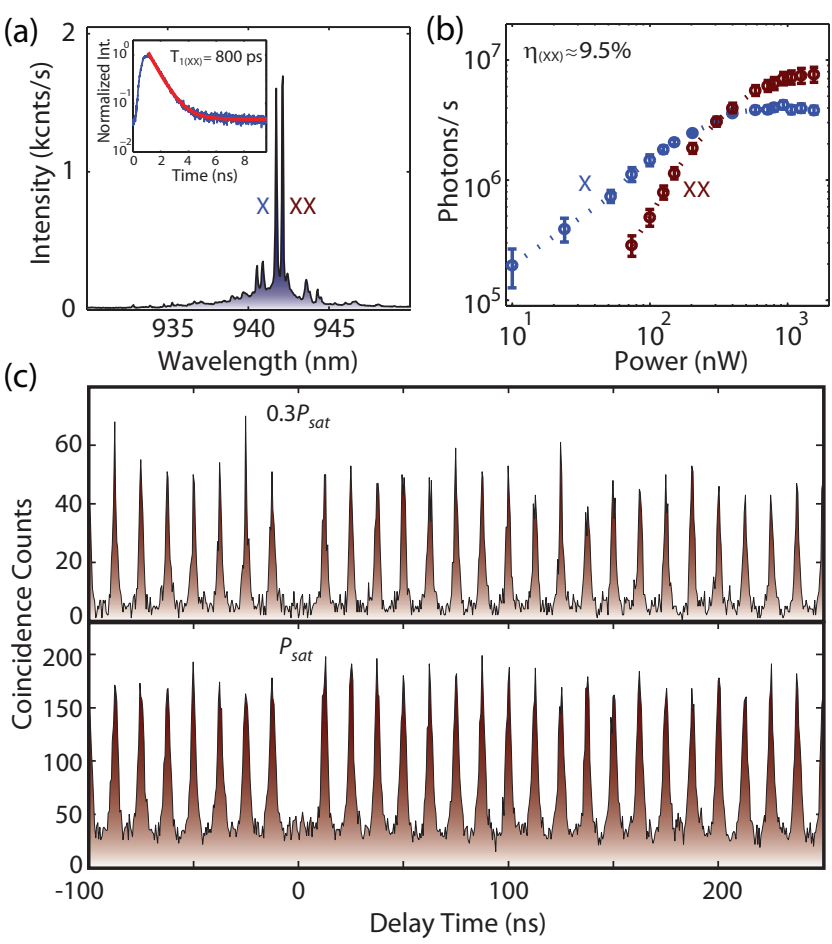

Fig. 6. Bright single-photon generation from a single QD coupled to the FM of the cavity. a) Low temperature $(\mathrm{T}=10 \mathrm{~K}) \mu$-PL spectrum of device BE2. Two bright emission lines are visible and labeled as exciton $(\mathrm{X})$ and bi-exciton (XX) based on their excitation power dependence as shown in b). inset: Timeresolved PL measurement on XX emission line, which reveals $T_{1}=800 \mathrm{ps}$. c) Demonstration of single-photon generation from the $\mathrm{XX}$ emission under low power $P=0.3 P_{\text {sat }}$ (upper panel) and high power $P=P_{\text {sat }}$ (lower panel) conditions.

on top of the FM of the cavity around $942 \mathrm{~nm}$. Fig. G(b) depicts the output intensity of $\mathrm{X}$ and $\mathrm{XX}$ emission lines as a function of excitation power in a double-logarithmic plot [28]. As is seen clearly, the $\mathrm{X}$ line has a linear power dependence while the XX line shows a quadratic increase with power as expected for exciton and bi-exciton emission from a single QD, respectively [29]. The output intensity of the $\mathrm{X}$ and $\mathrm{XX}$ emission lines in the figure are given as number of photons collected at the microscope objective, thus providing a direct estimate of total collection efficiency of the corresponding emission. Conversion of the integrated intensity of CCD counts to number of photons was done by measuring the total transmission of the optical path, as discussed in detail in the supplementary part of [17]. The extraction efficiency of exciton and bi-exciton emission are estimated as $5.4 \% \pm 0.4 \%$ and $9.5 \% \pm 1.4 \%$, respectively, by comparing the number of photons collected at the saturation power to the total number of photons generated from the corresponding emission line, assuming $100 \%$ radiative efficiency of the QD.

Having shown photon collection efficiencies on par with those seen in device BE1, we also performed autocorrelation measurements on the $\mathrm{XX}$ line under weak $\left(P=0.3 P_{\text {Sat }}\right)$ and strong excitation powers $\left(P=P_{S a t}\right)$, the results of which are shown in Fig. 6(c). Almost complete suppression of the $\tau=0$ peak is observed even at the saturation power, yielding a great improvement compared to the results obtained from device BE1. The constant background observed in the autocorrelation 

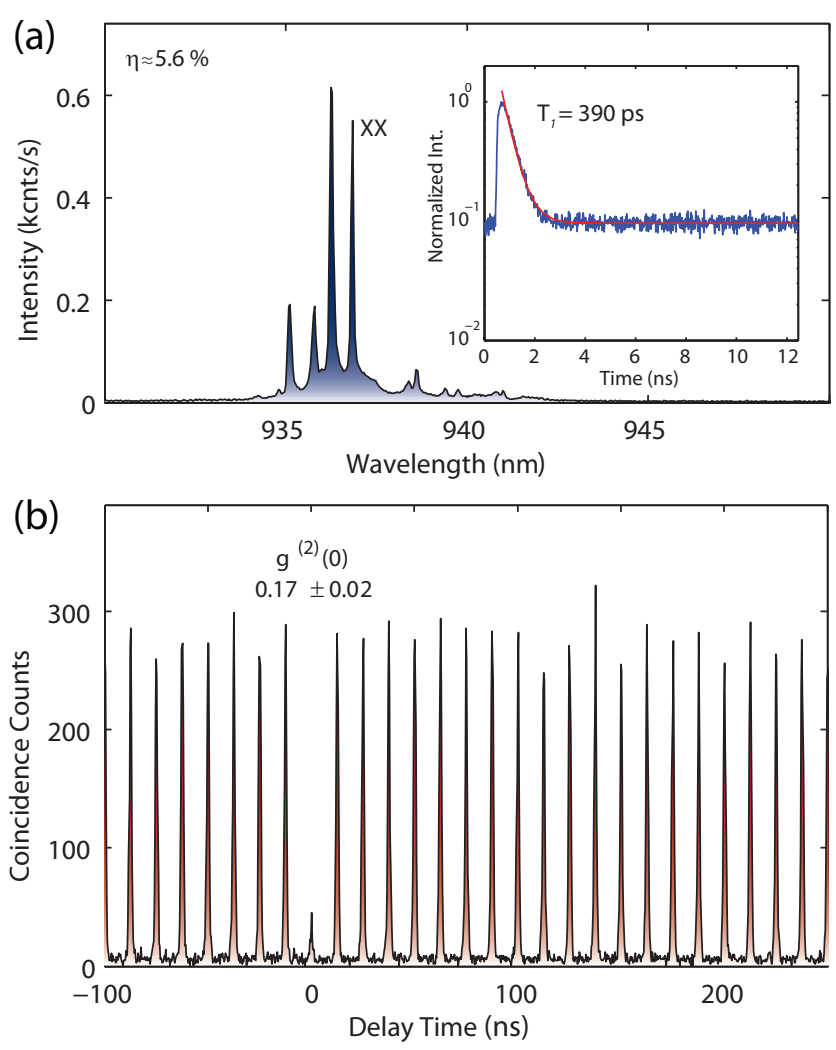

Fig. 7. Purcell-enhanced bright single-photon generation from a single QD. (a) Low temperature $(\mathrm{T}=10 \mathrm{~K}) \mu$-PL spectrum of device BE3, which shows sharp emission lines around $936 \mathrm{~nm}$ within the spectral width of the fundamental mode of the bullseye. inset: Time-resolved PL on QD emission at $937 \mathrm{~nm}$. (b) The result of an autocorrelation measurement performed on $\mathrm{XX}$ line under close-to-saturation excitation power.

measurements is mainly due to the overlap of broad correlation peaks indicating long lifetime of the measured emission. The inset of figure 6 (a) shows a direct measure of the lifetime of the XX emission through time-resolved PL setup. Applying an exponential fit to the measured decay curve gives the radiative lifetime as $T_{1}=800 \pm 70 \mathrm{ps}$, slightly more than a factor of two larger than that measured for device BE1.

The results obtained from BE2 showed a clear improvement in the purity of the single photons generated from a single $\mathrm{QD}$, with a cost of a longer radiative lifetime. A compromise between these two important parameters can also be achieved. Figure 7 a) shows a low temperature $\mu$-PL spectrum of another device, named BE3, under weak pulsed excitation $\left(\lambda_{E x c}=820 \mathrm{~nm}\right)$. Like the other devices, bright emission lines are visible on top of the cavity mode around $936 \mathrm{~nm}$, and are collected with an efficiency of $5.6 \% \pm 0.4 \%$ at saturation (not shown). The inset of Fig 7 (a) depicts the result of a time-revolved PL measurement performed on the XX line, which dominates the spectrum at elevated powers, and a radiative lifetime of about $390 \pm 15$ ps is estimated from the bi-exponential fit. This radiative lifetime is close to that measured for device BE1 and about a factor of two faster than that for BE2. In order to verify the single-photon nature of the measured emission line, an autocorrelation measurement was performed, the result of which is shown in Fig. 7b). The reduced area of the peak at zero time delay indicates a clear photon antibunching with a value of $g^{(2)}(\tau)=0.17<0.5$, thus proving that the source is dominantly composed of single photons with a multi-photon probability in-between that of devices BE1 and BE2.

Devices BE1, BE2, and BE3 show radiative lifetimes of $360 \mathrm{ps}, 800 \mathrm{ps}$, and $390 \mathrm{ps}$, indicating that the relative level of Purcell enhancement varies by a factor of 2.2 between the different devices. An estimate of the absolute Purcell enhancement factor $F_{p}$ requires a measurement of the QD lifetime without modification by the cavity. Unfortunately, the broad spectral bandwidth of the bullseye eliminates the potential for using temperature tuning to shift the QD lines offresonance from the cavity. We instead measured the lifetime of QDs within suspended waveguides [14] made from the same wafer, where we saw characteristic lifetimes between $1.4 \mathrm{~ns}$ and $1.6 \mathrm{~ns}$ for the neutral exciton (X) state (no Purcell enhancement is expected in these devices). However, as described above, the lifetime and $g^{(2)}(\tau)$ measurements for the bullseye devices were typically performed on the biexciton (XX) state, though we note that the collection efficiency of the exciton state was often equally as high. While the XX lifetime is in principle a factor of two times shorter than the $\mathrm{X}$ lifetime, other works [30], [31] have suggested that quantum confinement properties specific to the particular QD geometry may limit this reduction to a factor closer to 1.4. Taking this into account, we roughly estimate that a Purcell factor as high as $\approx 3$ has been demonstrated in these devices.

\section{Coherence time}

For quantum information processing applications, it is important not only to suppress multi-photon events but also to have the single photon wavepackets be indistinguishable [32], [16]. For an ideal transform-limited QD line, the coherence time $T_{2}$ would be twice the lifetime $T_{1}$, and values approaching this limit have been demonstrated [33], [34]. In practice, achieving such coherence times depends strongly on parameters such as temperature and the method by which the QD is optically excited (e.g., above-band vs. resonant excitation).

We investigate the coherence properties of the bright emission lines from all three bullseye devices by measuring the first-order field correlation function $g^{(1)}(\tau)$, which provides a direct access to the coherence time of the emission through [35]:

$$
\tau_{c}=\int_{-\infty}^{\infty}\left|g^{(1)}(\tau)\right|^{2} d \tau
$$

Experimentally, the $g^{(1)}(\tau)$ function is measured from the visibility of the interference fringes observed at the output port of the Michelson interferometer (Fig. 3 d)) according to:

$$
V(\tau)=\frac{\left(I_{\max }-I_{\min }\right)}{\left(I_{\max }+I_{\min }\right)}=\left|g^{(1)}(\tau)\right|
$$

for equal intensities of interfered light. The inset to figure 8 shows an example of high-resolution interference fringes of the 


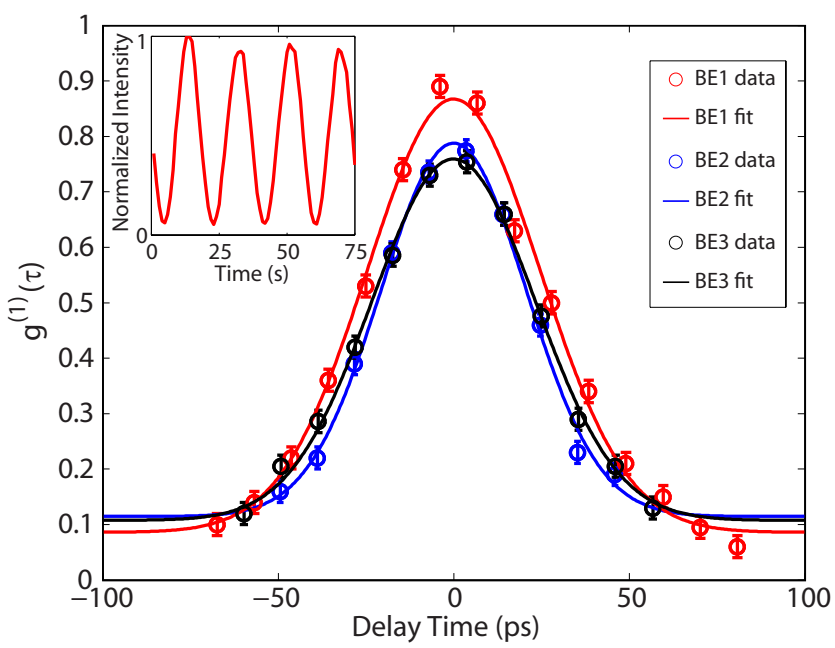

Fig. 8. The results of first-order field correlation function measurements on all three devices under low power above-band excitation conditions. The inset shows an example of the interference fringes produced at a position close to zero delay line in the Michelson interferometer.

XX emission line from device BE1 obtained under above-band excitation conditions $\left(\lambda_{E x c}=780 \mathrm{~nm}, \mathrm{CW}\right)$. The visibility of the fringes at each systematically varied time delay between the interferometer arms are calculated according to Eqn. 2] and plotted in Fig. 8 for all devices [36]. The visibility of the interference fringes decreases with an increase in delay as a result of the limited coherence time $\tau_{c}$ of the emission. Applying a Gaussian fit to the visibility data reveals $\tau_{c}=27 \mathrm{ps}$ $(\Gamma=100 \mu \mathrm{eV})$ for BE1 and $17 \mathrm{ps}(160 \mu \mathrm{eV})$ for BE2 and BE3. The emission linewidth estimated from $g^{(1)}(\tau)$ measurements is in full agreement with the linewidth obtained from the corresponding $\mu$-PL spectrum for BE2 and BE3. However, the emission spectrum of device BE1 revealed a linewidth of $130 \mu \mathrm{eV}$, which is broader than the linewidth obtained from $g^{(1)}(\tau)$ measurement $(100 \mu \mathrm{eV})$. The difference mainly arises from the limited spectral resolution of the $\mu$-PL setup estimated as $80 \mu \mathrm{eV}$ by measuring a narrow $\mathrm{CW}$ laser. The measured linewidths of emission from all devices are much broader than the reported homogenous linewidth of QDs that are nearly transform-limited $(\approx 1 \mu \mathrm{eV}$ to $2 \mu \mathrm{eV})$ [33], [34]. The main reason for this is thought to be the incoherent nature of the above-band excitation process, which excites several QDs within the excitation spot and thus enhances the dephasing processes due to the carrier-carrier interactions. In addition to that, spectral diffusion of the emission frequency due to fluctuating charges in the vicinity of the QDs may result in a broadening of the emission [37].

\section{DISCUSSION}

The results presented above can be further interpreted with the help of numerical simulations. In particular, we try to understand how the specific device geometry influences the aforementioned trade-offs in different performance characteristics, as well as the reduced collection efficiency and rate enhancement observed in experiments relative to the maximum predicted theoretical values in ref. [17].
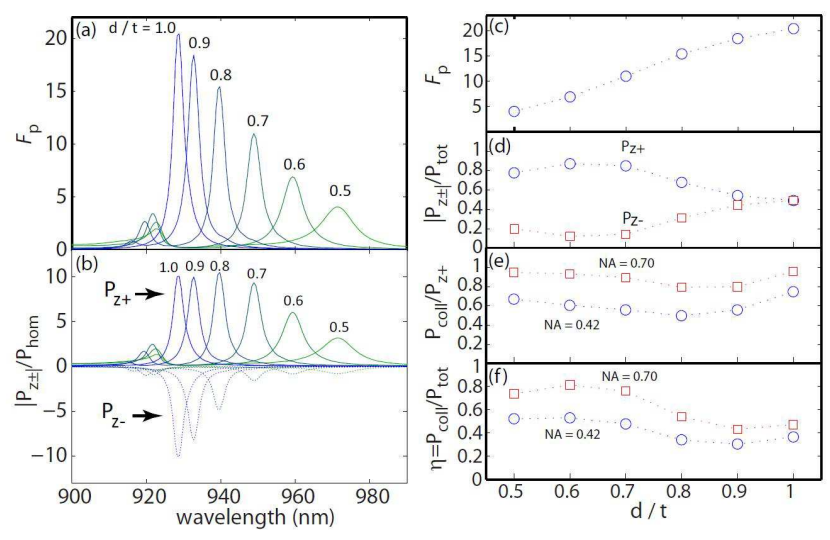

Fig. 9. (a) Purcell enhancement factor $F_{p}$ as a function of wavelength for various normalized trench depths $d / t$. (b) Ratio of the vertically emitted power in the $\pm z$ direction $\left(P_{z \pm}\right)$ to the total emitted power of a dipole in bulk GaAs $\left(P_{\text {hom }}\right)$, as a function of wavelength for various values of $d / t$. (c) $F_{p}$ as a function of $d / t$. (d) percentage of total power emitted upwards and downwards, as a function of $d / t$. (e) percentage of upwards emitted power collected by NA $=0.42 \mathrm{NA}=0.7$ lenses. (f) percentage of total emitted power collected by $\mathrm{NA}=0.42 \mathrm{NA}=0.7$ lenses.

\section{A. Tradeoffs in Purcell enhancement and collection efficiency}

We next illustrate the effects of varying trench depths on emission properties of the circular dielectric grating. The first key quantity of interest is the Purcell enhancement factor $F_{p}$. It is determined from simulation by the quantities $P_{\text {tot }}$ and $P_{\text {hom. }} . P_{\text {tot }}$ is the total power emitted by a dipole in the bullseye structure, while $P_{\text {hom }}$ is the power emitted by a dipole in a homogenous GaAs medium, so that $F_{p}$ is given by their ratio $P_{\text {tot }} / P_{\text {hom }}[25]$. Figures $\left[\right.$ a) and (c) show $F_{p}$ as a function of wavelength and normalized trench depth $d / t$. $F_{p}$ increases as $d / t$ approaches unity, with deeper trenches providing increased field confinement and higher cavity $Q$. This is a consequence of better overlap of the guided field inside the slab and the etched region, which leads to increased guided wave reflectivity and reduces coupling to out-of-plane radiation. The higher cavity $Q$ with increasing trench depth is seen in the decreasing resonance width in Fig. 9 (a); in addition, a strong blueshift of the cavity mode is observed.

The increase in $F_{p}$ with trench depth comes at a cost, however, as the fraction of light emitted above the structure $(+z$ direction) is reduced. Intuitively, this makes sense in that, once the grating is completely symmetric $(d / t=1$, for which $F_{p}$ is maximized), equal emission in the $+z$ and $-z$ directions should be expected. Figures 9 (b) and (d) show the upwards $\left(P_{\mathrm{z}}\right)$ and downwards $\left(P_{\mathrm{z}-}\right)$ extracted powers as a function of wavelength and normalized trench depth $d / t$. Asymmetric and preferential upwards emission (determined by the ratio $P_{z^{+}} / P_{z^{-}}$) is maximized with $d / t=0.6$, for which $P_{z+} / P_{z^{-}}=7.1$, but comes at the expense of a significantly reduced $F_{p}$ of 6.9 in comparison to its peak value of nearly 20.

For useful single photon emission, preferential upwards emission alone is not sufficient. We also require the emission to be directed within a relatively narrow divergence angle farfield pattern, so that the majority of this emission can be collected by a standard focusing optic. Figure 9(e) shows the 
fraction of upwards emission that is collected $\left(P_{\text {coll }} / P_{\mathrm{z}+}\right)$ by $\mathrm{NA}=0.42$ and $\mathrm{NA}=0.7$ optics, at the peak emission wavelength for each $d / t$ (see Fig. 9a)). We note that this value is close to unity for the NA=0.7 optic, indicating a strong level of directionality, which we also expect based on the farfield emission pattern in Fig. 2 2 d). In addition, we see that the collection of the upwards-emitted power can vary by as much as $33 \%$ for the trench depth range considered. Finally, Fig. 9 f $)$ shows the overall collection efficiency $\eta\left(=P_{\text {coll }} / P_{\text {tot }}\right)$ as a function of trench depth, which is the quantity that is actually measured in the experiments of the previous section. $\eta$ peaks at a value of $\approx 80 \%$ for a NA $=0.7$ optic, while for the $\mathrm{NA}=0.42$ optic used in our experiments, this value is $\approx 50 \%$.

It is evident from these results that sufficiently accurate control of the trench depth is a necessity, not only for optimal spectral alignment, but also for optimal extraction efficiency. For instance, for a trench depth $d=0.8 t$, the collection efficiency into a 0.42 NA objective drops to approximately $34 \%$ from the $53 \%$ for $d=0.6 t$. As $t=190 \mathrm{~nm}$, a $40 \mathrm{~nm}$ difference in trench depth, which we might expect to observe based on the tolerances of our fabrication process, can lower the maximum extraction efficiency significantly.

Along with radiative lifetime (determined by $F_{p}$ ) and collection efficiency, the third key characteristic of the devices measured in the previous section is the single photon purity (i.e., the value of $g^{(2)}(0)$ ). Our simulations do not directly address the effects which cause $g^{(2)}(0)>0$. However, many works [18], [19] have attributed this to cavity-enhanced outcoupling of multi-excitonic and hybridized QD-wetting layer states that are spectrally resonant with the QD state of interest. In such a scenario, strongly Purcell-enhanced devices are likely to have $g^{(2)}(0)$ levels that significantly differ from zero, while devices lacking Purcell enhancement can show $g^{(2)}(0) \approx$ 0 even under above-band pumping near saturation [13]. From this, we speculate that shallower grating depths $(0.5<d / t<$ 0.6 ), which lead to smaller $F_{p}$ in Fig. 10 (c), may also lead to enhanced emission purity. Furthermore, this benefit would come together with increased collection efficiency (Fig. 10(f)) as a consequence of improved vertical extraction and emission asymmetry (Fig. 10/d)).

\section{B. Influence of Dipole Position and Orientation}

Other important factors affecting both the achieved Purcell radiative rate enhancement and overall photon collection efficiency are dipole position and orientation within the nanostructure. Considering the fundamental mode of the BE cavity in Fig.2 (shown also in Fig.10 b) and (c)), which we label as $M_{0}$, we note that due to the circular symmetry of the nanostructure, both mode $M_{0}$ and a degenerate, $90^{\circ}$ version of it (henceforth referred to as $\mathbf{E}_{1}$ and $\mathbf{E}_{\mathbf{2}}$, respectively) are simultaneously supported. Mode $\mathbf{E}_{\mathbf{1}}$ is exclusively excited by azimuthally oriented dipoles along the radial direction $(\mathbf{r})$, while mode $\mathbf{E}_{\mathbf{2}}$ is exclusively excited by radially oriented dipoles along r. The specific instances of the modes excited by $x$-polarized and $y$-polarized dipoles are shown in Fig. 10 (b) and (c), and a dipole oriented at an arbitrary angle in the plane would then be expected to radiate into both of these modes.

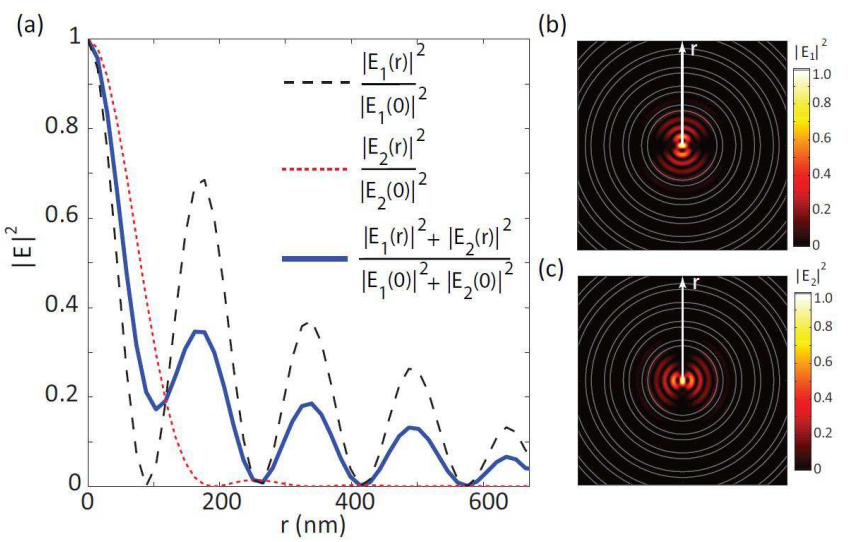

Fig. 10. (a) Electric field squared of first order modes $\mathbf{E}_{\mathbf{1}}(\mathbf{r})$ and $\mathbf{E}_{\mathbf{2}}(\mathbf{r})$, with $r$ the distance along the radial direction. Continuous line: sum of squared electric fields for modes $\mathbf{E}_{\mathbf{1}}$ and $\mathbf{E}_{\mathbf{2}}$. Field profiles for modes $\mathbf{E}_{\mathbf{1}}(\mathbf{r})$ and $\mathbf{E}_{\mathbf{2}}(\mathbf{r})$, which are excited by (b) $x$-polarized and (c) $y$-polarized dipoles, respectively.

Figure 10(a) shows the intensities of $\left|\mathbf{E}_{\mathbf{1}}\right|^{2}$ and $\left|\mathbf{E}_{\mathbf{2}}\right|^{2}$ as functions of position $r$ along the radial direction (in this case, the $y$ direction), relative to those at the center. Since the radiative rate into a particular mode is proportional to the local modal electric field squared [25], it is apparent that Purcell radiative rate enhancement can vary significantly over very small position ranges, and also with dipole orientation. For a dipole with equal radial and azimuthal components, the plot of $|\mathbf{E}(r)|^{2}=\left|\mathbf{E}_{\mathbf{1}}(\mathbf{r})\right|^{2}+\left|\mathbf{E}_{\mathbf{2}}(r)\right|^{2}$ shown in Fig 10(a) makes it apparent that positioning within $\mathrm{a} \approx 200 \mathrm{~nm}$ radius from the center is necessary for maximal modal coupling and Purcell enhancement to be achieved.

Coupling to both of the degenerate $M_{0}$ modes is not the only potential source of non-ideality for dipoles located away from the center of the cavity. Due to its large dimensions, the cavity supports not only the mode shown in Fig. 2 a) and (b), but also an ensemble of broad, spectrally overlapping resonances that can be excited by dipoles offset from the bullseye center. These resonances can have $\beta$-factors comparable to that of the main mode (depending on the dipole location), however less directional far-fields. In this situation, an enhanced spontaneous emission rate can be achieved (with contributions from all modes), together with a reduced overall collection efficiency. To illustrate, we show in Fig. 111(a) the field profile of a high order mode that is excited on the same grating as in Fig. 2 2 a), when an $x$-oriented dipole is placed at a distance of $260 \mathrm{~nm}$ away from the center. As shown in Fig. 11 b), this broad mode $\left(M_{1}\right)$ is centered at $957 \mathrm{~nm}$, and overlaps with the main cavity mode $M_{0}$ at $949 \mathrm{~nm}$ (compare with $P_{z+}$ spectrum obtained for a centered dipole shown by the dotted line). The far-field of mode $M_{1}$ is such that only $\approx 26 \%$ of the total upwards radiated power can be collected by an $\mathrm{NA}=0.42$ objective, significantly smaller than the $\approx 56 \%$ for $M_{0}$. It is also worthwhile noticing in Fig. 11 b) that the radiative rates for both modes $\mathrm{M}_{0}$ and $\mathrm{M}_{1}$ for the offset dipole case are considerably lower than for a centered dipole (dotted line). This is expected because the modified radiative rate for a dipole in the nanostructure is proportional to $\left|\mathbf{E}\left(\mathbf{r}_{\mathbf{0}}\right)\right|^{2}$, where $\mathbf{E}\left(\mathbf{r}_{\mathbf{0}}\right)$ is the electric field at the dipole position and for both 

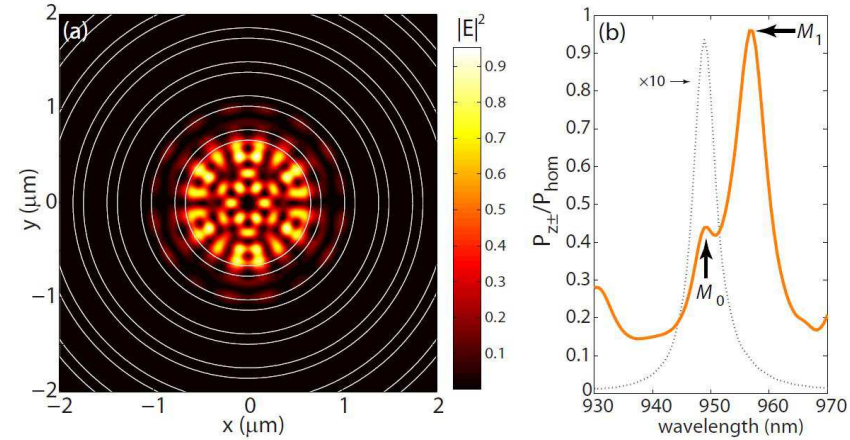

Fig. 11. (a) Field profile of a higher order mode of the structure in Fig. 22 a), excited by a horizontally oriented dipole located $260 \mathrm{~nm}$ away from the center. (b) Upwards-emitted power, normalized to the homogeneous medium dipole radiated power, for the offset dipole. Dotted line: same, for dipole at the center; note the 10x scaling between the two curves.

modes the offset dipole is located near a null.

\section{Quasi-Resonant Excitation}

The coherence properties of single QD emission can be improved under selective excitation conditions, where the laser energy is tuned to a higher energy state of an individual QD. This so called "quasi-resonant" excitation process limits the number of excited QDs, thus reducing the dephasing processes causing a linewidth broadening as mentioned before. Moreover, it also reduces the multi-photon generation probability significantly because of a reduced probability of creating more than one electron-hole pair in the QD. Figures 12 a) and (b) show emission spectra of device BE1 taken under quasi-resonant excitation, where the pulsed laser is tuned to $863.5 \mathrm{~nm}$ and $894.2 \mathrm{~nm}$, respectively. In each spectrum, a bright excitonic emission line is visible at $937 \mathrm{~nm}$, on which an autocorrelation measurement was also performed to investigate its single emitter nature. The result of the experiments are depicted in Figures 12 (c) and (d). A complete missing peak observed at zero time delay in both figures proves that the applied excitation scheme resulted in almost perfect singlephoton generation from the measured emission line. An interesting feature seen in the figures is that the correlation peaks close to zero delay are much larger than the outer side peaks. Similar results were reported under quasi-resonant excitation of a single QD [38] and the observed blinking effect on a long time scale was attributed to a charge fluctuation of the QD. The linewidth of the measured state is extracted as $\Gamma=100 \mu \mathrm{eV}$ from the corresponding $\mu$-PL spectrum, which is narrower than the typical linewidths measured in $\mu$-PL measurements under above-band excitation conditions.

\section{CONCLUSiOns}

We have presented a series of measurements of single quantum dots in circular Bragg grating 'bullseye' microcavities. These devices, first presented in ref. [17], offer a combination of features valuable to single photon sources: high extraction efficiency, moderate spectral bandwidth, and radiative rate enhancement, all within a planar geometry (a)
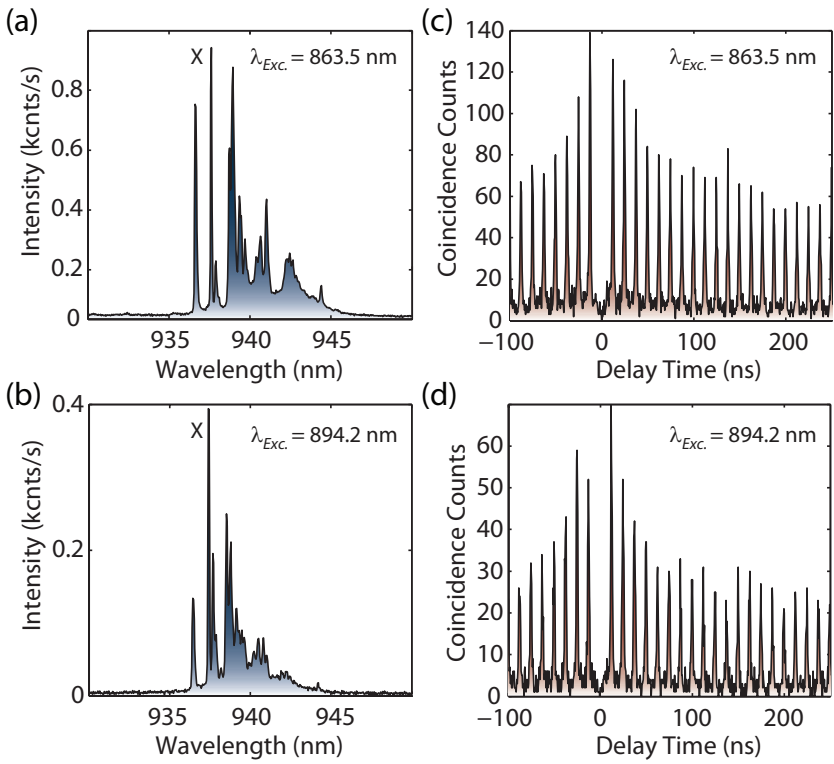

Fig. 12. Higher-energy state excitation of a single QD in device BE1. (a) Emission spectrum of device BE1 under quasi-resonant excitation with $\lambda_{E x c .}=863.5 \mathrm{~nm}$ and (c) measured second-order correlation function of excitonic emission line $\mathrm{X}$ at $937 \mathrm{~nm}$. The vanished peak at zero time delay indicates a strong suppression of multi-photon emission probability. (b) Emission spectrum of BE1 with $\lambda_{E x c}=894.2 \mathrm{~nm}$ and (d) the measured second-order correlation function of the same peak.

requiring a relatively straightforward fabrication procedure. Here, we focus on the tradeoff between rate enhancement and multi-photon probability present in these devices under aboveband optical pumping. We first demonstrate that the brightest devices studied in ref. [17] indeed show antibunched photon statistics, but that the multi-photon probability is somewhat high (though still low enough that the source is dominantly comprised of single photons). We then consider two other devices in which the collection efficiency remains high, but the multi-photon probability is reduced, albeit at the expense of a longer radiative rate. We find that a near-complete suppression of multi-photon probability is possible in devices with high collection efficiency, but that the rate enhancement is correspondingly the lowest we see. These results suggest that unwanted background radiation (for instance, from transitions of multi-excitonic or hybrid quantum well-QD states) is enhanced and funneled through the cavity, leading to less pure single photon emission [18], [19]. Cavities with reduced Purcell enhancement may thus lead to reduced multi-photon emission [13].

Numerical simulations were performed to help interpret the physical scenarios that lead to the aforementioned compromises and the reduced collection efficiency and rate enhancement values observed relative to the maximum predicted values. The most straightforward method to improving the observed collection efficiency is through using an increased numerical aperture collection optic. Moving to an NA $=0.7$ optic from the current NA $=0.42$ optic is predicted to improve the collection efficiency for existing devices by $\approx 60 \%$. Beyond this, precise control of the device geometry, both in terms of the etched trench depth and the QD position [20], are needed 
to achieve the desired combination of rate enhancement and collection efficiency. In particular, shallower grating trenches lead to increased light outcoupling, as well as a more highly asymmetric vertical emission, which ultimately leads to higher collection efficiencies. This comes at the cost of lower Purcell enhancement, which however may potentially lead to a more pure single photon emission, as discussed above.

Ideally, the bullseye cavity would be useful not only as a bright single photon source but as a bright source of indistinguishable single photons. Measurements under above-bandgap excitation indicate that the QD coherence time is extremely short ( $<30 \mathrm{ps})$, so that quasi-resonant excitation [16], [34] or post emission spectral/temporal filtering [39], [40] will be required to generate indistinguishable photons. As a preliminary step towards this, we present photoluminescence spectra and photon correlation measurements of devices optically pumped at a wavelength resonant with higher states of the QD and/or cavity, for which near-complete suppression of multi-photon events is observed. Such quasi-resonant excitation may be an eventual route to simultaneously achieving high collection efficiency, strong rate enhancement, and suppressed multiphoton probability.

\section{ACKNOWLEDGEMENTS}

This work has been partly supported by the NISTCNST/UMD-NanoCenter Cooperative Agreement. We thank Nick Bertone from OptoElectronic Components for loan of a high timing resolution single photon detector. The identification of any commercial product or trade name does not imply endorsement or recommendation by the National Institute of Standards and Technology.

\section{REFERENCES}

[1] A. J. Shields, "Semiconductor quantum light sources," Nature Photonics, vol. 1, pp. 215-223, Apr. 2007.

[2] P. Michler, A. Kiraz, C. Becher, W. V. Schoenfeld, P. M. Petroff, L. Zhang, E. Hu, and A. Imamoglu, "A Quantum Dot Single-Photon Turnstile Device," Science, vol. 290, pp. 2282-2285, Dec. 2000.

[3] C. Santori, M. Pelton, G. Solomon, Y. Dale, and Y. Yamamoto, "Triggered Single Photons from a Quantum Dot," Phys. Rev. Lett., vol. 86, no. 8, pp. 1502-1505, Feb. 2001

[4] J.-M. Gérard and B. Gayral, "Strong Purcell effect for InAs quantum boxes in three-dimensional solid-state microcavities," J. Lightwave Tech. vol. 17, no. 11, pp. 2089-2095, Nov. 1999.

[5] H. Benisty, H. D. Neve, and C. Weisbuch, "Impact of Planar Microcavity Effects on Light Extraction - Part I: Basic Concepts and Analytical Trends," IEEE J. Quan. Elec., vol. 34, no. 9, pp. 1612-1631, Sep. 1998.

[6] W. L. Barnes, G. Björk, J. Gérard, P. Jonsson, J. A. E. Wasey, P. T. Worthing, and V. Zwiller, "Solid-state single photon sources: light collection strategies," Eur. Phys. J. D, vol. 18, pp. 197-210, 2002.

[7] J.-M. Gérard and B. Gayral, "Towards high-efficiency quantum-dot single photon sources," Proceedings of SPIE, vol. 5361, pp. 88-95, 2004.

[8] V. Zwiller and G. Bjork, "Improved light extraction from emitters in high refractive index materials using solid immersion lenses," J. Appl. Phys., vol. 92, pp. 660-665, Jul. 2002.

[9] A. N. Vamivakas, M. Atature, J. Dreiser, S. T. Yilmaz, A. Badolato, A. K. Swan, B. B. Goldberg, A. Imamoglu, and M. S. Unlu, "Strong extinction of a far-field laser beam by a single quantum dot," Nano Letters, vol. 7, no. 9, pp. 2892-2896, 2007.

[10] S. Strauf, N. G. Stoltz, M. T. Rakher, L. A. Coldren, P. M. Petroff, and D. Bouwmeester, "High-frequency single-photon source with polarization control," Nature Photonics, vol. 1, pp. 704-708, Dec. 2007.

[11] G. Solomon, M. Pelton, and Y. Yamamoto, "Single-mode spontaneous emission from a single quantum dot in a three-dimensional microcavity," Phys. Rev. Lett., vol. 86, no. 17, pp. 3903-3906, 2001.
[12] M. Toishi, D. Englund, A. Faraon, and J. Vuckovic, "High-brightness single photon source from a quantum dot in a directional-emission nanocavity," Opt. Express, vol. 17, no. 7, pp. 14618-14 626, 2009.

[13] J. Claudon, J. Bleuse, N. S. Malik, M. Bazin, P. Jaffrennou, N. Gregersen, C. Sauvan, P. Lalanne, and J. Gérard, "A highly efficient single-photon source based on a quantum dot in a photonic nanowire," Nature Photonics, vol. 4, pp. 174-177, Mar. 2010.

[14] M. Davanço, M. T. Rakher, , W. Wegscheider, D. Schuh, A. Badolato, and K. Srinivasan, "Efficient quantum dot single photon extraction into an optical fiber using a nanophotonic directional coupler," Appl. Phys. Lett., vol. 99, p. 121101, 2011.

[15] S. Varoutsis, S. Laurent, P. Kramper, A. Lemaître, I. Sagnes, I. RobertPhilip, and I. Abram, "Restoration of photon indistinguishability in the emission of a semiconductor quantum dot," Phys. Rev. B, vol. 72, no. 4, p. 041303, Jul 2005.

[16] C. Santori, D. Fattal, J. Vuckovic, G. Solomon, and Y. Yamamoto, "Indistinguishable photons from a single-photon device," Nature, vol. 419, pp. 594-597, Oct. 2002.

[17] M. Davanço, M. T. Rakher, D. Schuh, A. Badolato, and K. Srinivasan, "A circular dielectric grating for vertical extraction of single quantum dot emission," Appl. Phys. Lett., vol. 99, p. 041102, 2011.

[18] M. Winger, T. Volz, G. Tarel, S. Portolan, A. Badolato, K. Hennessy, E. L. Hu, A. Beveratos, J. Finley, V. Savona, and A. Imamoglu, "Explanation of Photon Correlations in the Far-Off-Resonance Optical Emission from a Quantum - Dot Cavity System," Phys. Rev. Lett., vol. 103, p. 207403, 2009.

[19] A. Laucht, M. Kaniber, A. Mohtashami, N. Hauke, M. Michler, and J. Finley, "Temporal monitoring of nonresonant feeding of semiconductor nanocavity modes by quantum dot multiexciton transitions," Phys. Rev. B, vol. 81, p. 241302, Jun 2010.

[20] K. Hennessy, A. Badolato, M. Winger, D. Gerace, M. Atature, S. Guide, S. Falt, E. Hu, and A. Imamoglu, "Quantum nature of a strongly coupled single quantum dot-cavity system," Nature (London), vol. 445, pp. 896899, Feb. 2007.

[21] A. Hardy, D. Welch, and W. Streifer, "Analysis of second-order gratings," Quantum Electronics, IEEE Journal of, vol. 25, no. 10, pp. 2096 -2105 , oct 1989.

[22] D. Taillaert, W. Bogaerts, P. Bienstman, T. F. Krauss, P. V. Daele, I. Moerman, S. Vertuyft, K. D. Mesel, and R. Baets, "An Out-of-Plane Grating Coupler for Efficient Butt-Coupling Between Compact Panar Waveguides and Single-Mode Fibers," IEEE J. Quan. Elec., vol. 38, no. 7, pp. 949-955, Jul. 2002.

[23] M. Y. Su and R. P. Mirin, "Enhanced light extraction from circular bragg grating coupled microcavities," Applied Physics Letters, vol. 89, no. 3, p. 033105, 2006.

[24] W. M. J. Green, J. Scheuer, G. DeRose, and A. Yariv, "Vertically emitting annular Bragg lasers using polymer epitaxial transfer," Appl. Phys. Lett., vol. 85, no. 17, pp. 3669-3671, Oct. 2004.

[25] Y. Xu, J. S. Vučković, R. K. Lee, O. J. Painter, A. Scherer, and A. Yariv, "Finite-difference time-domain calculation of spontaneous emission lifetime in a microcavity," J. Opt. Soc. Am. B, vol. 16, no. 3, pp. $465-474,1999$.

[26] J. Vučković, O. Painter, Y. Xu, A. Yariv, and A. Scherer, "FDTD Calculation of the Spontaneous Emission Coupling Factor in Optical Microcavities," IEEE J. Quan. Elec., vol. 35, no. 8, pp. 1168-1175, Aug. 1999.

[27] The $g^{(2)}(0)$ value is determined by comparing the integrated area of the peak around time zero to the average area of the peaks away from time zero. The uncertainty on this value is given by the standard deviation in the area of the peaks away from time zero.

[28] The photon count rate vs. pump power plot in Fig. 6(b) is determined by fitting the spectral peak to a Lorentzian and integrating the area within the Lorentzian. Error bars are due to the uncertainty of the fit and are one standard deviation in amplitude.

[29] R. M. Thompson, R. M. Stevenson, A. J. Shields, I. Farrer, C. J. Lobo, D. A. Ritchie, M. L. Leadbeater, and M. Pepper, "Single-photon emission from exciton complexes in individual quantum dots," Phys. Rev. B, vol. 64, p. 201302(R), 2002.

[30] C. Santori, G. S. Solomon, M. Pelton, and Y. Yamamoto, "Time-resolved spectroscopy of multiexcitonic decay in an InAs quantum dot," Phys. Rev. B, vol. 65, p. 073310, Feb. 2002.

[31] A. Kiraz, S. Falth, C. Becher, B. Gayral, W. V. Schoenfeld, P. M. Petroff, L. Zhang, E. Hu, and A. Imamoglu, "Photon correlation spectroscopy of a single quantum dot," Phys. Rev. B, vol. 65, p. 161303, Mar. 2002.

[32] A. Kiraz, M. Atature, and A. Imamoglu, "Quantum-dot single-photon 
sources: Prospects for applications in linear optics quantum-information processing," Phys. Rev. A, vol. 69, p. 032305, 2004.

[33] M. Bayer and A. Forchel, "Temperature dependence of the exciton homogeneous linewidth in $\operatorname{In}_{0.60} \mathrm{Ga}_{0.40} \mathrm{As} / \mathrm{GaAs}$ self-assembled quantum dots," Phys. Rev. B, vol. 65, p. 041308(R), 2002.

[34] S. Ates, S. M. Ulrich, S. Reitzenstein, A. Löffler, A. Forchel, and P. Michler, "Post-selected indistinguishable photons from the resonance fluorescence of a single quantum dot in a microcavity," Phys. Rev. Lett. vol. 103, no. 16, p. 167402, Oct 2009.

[35] R. Loudon, The Quantum Theory of Light, 3rd ed. Oxford University Press, 2000

[36] The error bars in the $g^{(1)}(\tau)$ data in Fig. 8 are determined by the fluctuations in the inteference contrast and are one standard deviation in amplitude.

[37] J. Seufert, R. Weigand, G. Bacher, T. Kümmell, A. Forchel, K. Leonardi, and D. Hommel, "Spectral diffusion of the exciton transition in a single self-organized quantum dot," Appl. Phys. Lett., vol. 76, p. 1872, 2000.

[38] C. Santori, D. Fattal, J. Vuckovic, G. S. Solomon, E. Waks, and Y. Yamamoto, "Submicrosecond correlations in photoluminescence from InAs quantum dots," Phys. Rev. B, vol. 69, p. 205324, May 2004.

[39] R. B. Patel, A. J. Bennett, K. Cooper, P. Atkinson, C. A. Nicoll, D. A. Ritchie, and A. J. Shields, "Postselective two-photon interference from a continuous nonclassical stream of photons emitted by a quantum dot," Phys. Rev. Lett., vol. 100, no. 20, p. 207405, May 2008.

[40] M. T. Rakher and K. Srinivasan, "Subnanosecond electro-optic modulation of triggered single photons from a quantum dot," Appl. Phys. Lett., vol. 98, no. 21, p. 211103, May 2011. 